\title{
Root Canal Treatment on Vertucci Type V Configuration - A Case Report
}

\author{
Hernawatiningsih ${ }^{1}$, Yulita Kristanti ${ }^{1}$, Dayinah Harman Subandhi ${ }^{2}$

 \\ Dentistry, Gadjah Mada University \\ ${ }^{2}$ Department of Conservative Dentistry, Faculty Of Dentistry Gadjah Mada University \\ *Coresponding author email : hernawatiningsih@gmail.com \\ Authorsemail:litaugm17@gmail.com,dayharsoe@gmail.com
}

\begin{abstract}
Root canal configuration influence the succeed of root canal treatment, because the morphological complexity of the root canal makes the procedure for preparation and obturation of the root canal more challenging. The anatomy and morphology of the root canal can be classified according to the Vertucci root canal classification system. In the Vertucci type V classification at the apical third of root the canal is divided into two separate root canals with two different apical foramen. This condition requires appropriate obturation techniques in order to obtain a hermetic seal. Case report: A 19 year old female patient wanted her upper left posterior tooth to be treated. Post dental history revealed there was spontaneous pain in the tooth, particularly happened at night. There was no pain detected during examination. The objective examination showed there was secondary caries in mesioincisal area of maxillary left first premolar tooth. Percussion, palpation and thermal vitality test was negative. The periapical radiograph revealed a radiolucent area at the apex of the tooth. The diagnosis established for this tooth was partial pulp necrosis with asymptomatic apical periodontitis. The configuration of the root canal was established as Vertucci type V. Root canal treatment using rotary crown down preparation technique and obturation with warm vertical condensation were performed, followed by class II composite resin restoration reinforced by short fiber reinforced composite. Conclusion: Root canal treatment with warm vertical condensation obturation technique is the appropriate treatment of choice for Vertucci type $\mathrm{V}$ root canal configuration.
\end{abstract}

Keywords: Root canal treatment, Vertucci type $V$, Warm vertical condensation 


\section{INTRODUCTION}

The goal of root canal treatment is to clean thoroughly and produce a canal shape that can be fully obturated with inert filling materials in order to avoid the existence of the remaining necrotic tissue and to prevent the penetration of the bacteria to the monoblock system of the root canal. An accurate diagnosis, an appropriate treatment planning, an adequate debridement, sterilization and obturation are some prerequisite factors for a successful root canal treatment. [1]. The complex anatomy of the root canal should be thoroughly understood for proper management, better diagnosis that will lead to the successful root canal treatment [2].

Studies report that dental anatomy variations are influenced by several factors, namely: ethnic, age, and gender. The maxillary first premolars are the most difficult teeth to be treated. Some factors regarding these difficulties are the number of roots, number of root canals, direction of root canal, configuration of the pulp cavity, and difficulty in visualizing the apical dimension. Many previous studies have revealed the existence of morphological variations and the number of root canals in maxillary first premolars related with problems in root canal treatment [1]

Root canals variations can be classified according to the Vertucci root canal classification system. Vertucci Type $\mathrm{V}$ performs as a single root canal in the coronal part, but in the the apical third of root the canal is divided into two separate root canals with two different apical foramen apical [3]. This condition requires proper obturation techniques to achieve hermetic obturation hence increase the successful of root canal treatment. Some obturation techniques of root canal treatment are chosen depending on the root canal anatomy and the purpose of treatment in each case. The basic obturation techniques include lateral condensation and hot vertical condensation [4].

\section{CASE REPORT}

19-year-old female patient came to the Conservative Dentistry Clinic of Prof. Soedomo Dental Hospital Gadjah Mada University, wanted her upper left posterior tooth to be treated. The patient had tooth filling a year before the examination, and loose a month later. Patient has experienced spontaneous pain in the tooth, especially at night. There was no pain detected during examination. Objective examination showed there was secondary caries in the maxillary left first premolar tooth (Figure 1) Percussion, palpation and thermal vitality test was negative. Pain was occured during root canal exploration using smooth broach. Infiltration anesthesia in the mucobuccal and palatal folds of tooth 24 was performed with 4\% articaine solution and 1: 100,000 epinephrine (Septocaine, Septodont). The periapical radiograph revealed a radiolucent area at the apex of the tooth.

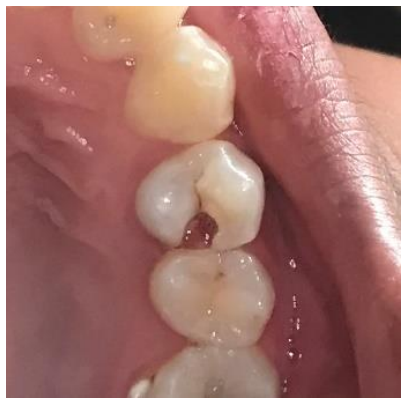

(A)

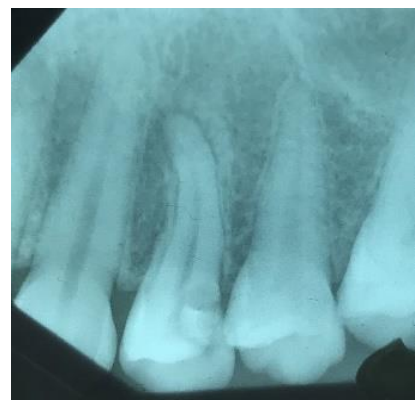

(B)

Figure 1. (A) Clinical features of tooth 24, appears cavity in the distoocclusal area of pulp depth; (B) Preoperative radiograph.

The diagnosis established for this tooth was partial pulp necrosis with asymptomatic apical periodontitis. One orifice was found in tooth 24 and while exploring the root canal using smooth broach, branching was found at the apical third, to the buccal and palatal direction (Vertucci type V). At the first visit, artificial walls were made using resin modified glass ionomer (Glass Ionomer Light Cured, GC) (Figure 2.A), and working length measurements were performed using K-file \# 15 in the buccal root canal, Hedstroem file \# 15 in the palatal root canal (Figure 2.B) The working length of the $19 \mathrm{~mm}$ buccal root canal and the $18 \mathrm{~mm}$ palatal root canal were obtained from the measurements that have been confirmed by the electronic apex locator and radiograph. Root canal preparation was carried out using the crown down technique using Protaper Next rotary file (Dentsply), 
interspersed with irrigation using $2.5 \% \mathrm{NaOCl}$ and saline. Root canal dressings use calcium hydroxide (UltraCal® XS, Ultradent).

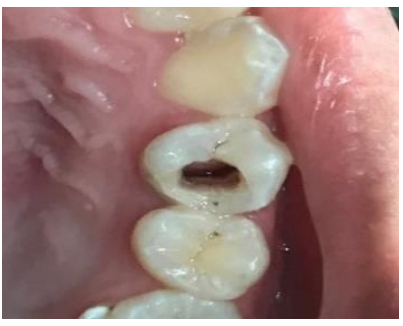

(A)

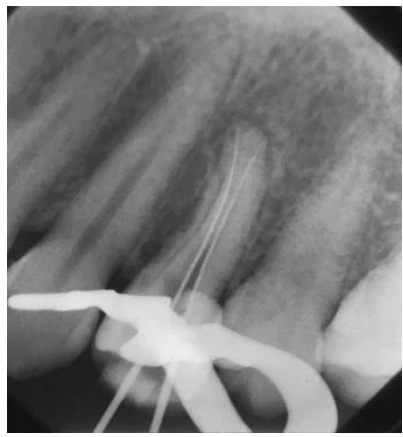

(B)

Figure 2. (A) Clinical features of tooth 24 after artificial wall were made; (B) Working length assessment of tooth 24 shows the tip of the file has reached apical constriction in the buccal and palatal.

On the second visit, after the X2 Protaper Next gutta percha fitting was suitable to the working length (Figure 3.A) then the root canal was oburated with warm vertical condensation technique using Gutta Percha Obturation System (Free Fill, Denjoy) (Figure 3.B). On the third visit, class II composite resin restorations with short fiber reinforced composite reinforcement was carried out (Figure 3.C). A week later the patient came for recall and no complaints were noted.

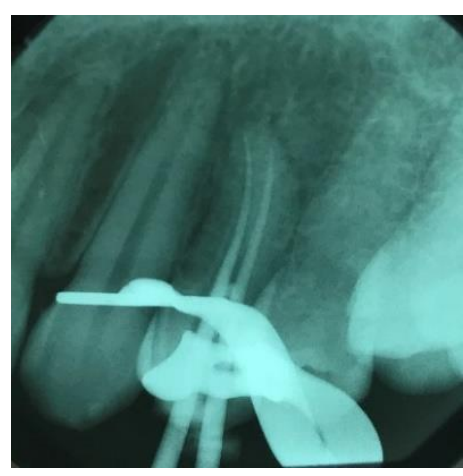

(A)

(A)

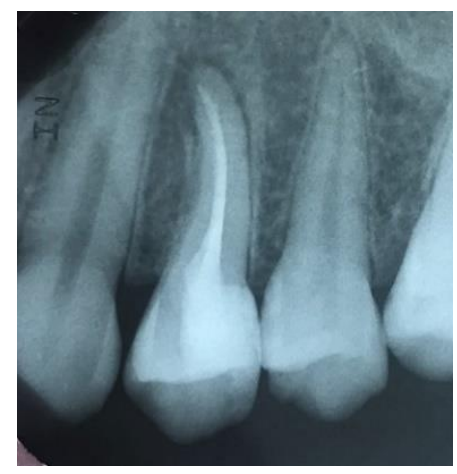

(B)

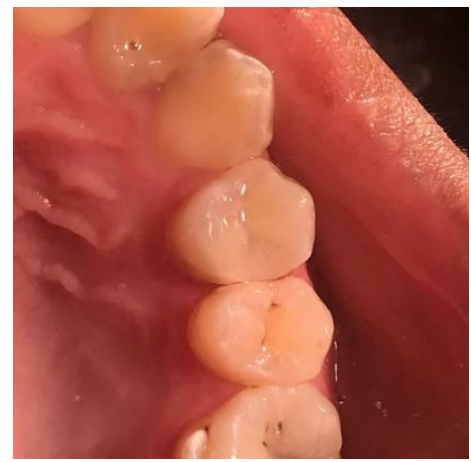

(C)

Figure 3. (A) Gutta percha fitting; (B) Radiograph after obturation; (C) Clinical features after restoration.

\section{DISCUSSION}

To support the successful of root canal treatment tooth with Vertucci type V root canal configuration, precise identification and valid morphological understanding are needed. This is because preparation and obturation in such cases is difficult and challenging. The maxillary first premolar tooth has a 
variety of root canal system and some previous study stated that it was the most complex teeth in root and canal morphology. The incidence of maxillary first premolars with one root varies from $30 \%$ to $60 \%$; two roots, 41.7 to $70 \%$; and three roots, 0 to $6 \%$. In addition, the occurrence of two canals in these teeth ranged from $73.3 \%$ to $92 \%$ in different morphology studies [1].

Vertucci (2005) classified morphological patterns of the root canal systems into eight types: (1) type I: a single canal present from the pulp chamber to the apex; (2) type II: 2 separate canals leave the pulp chamber but join to form one canal to the site of exiting; (3) type III: one canal leaves the pulp chamber, divides into two within the root, and then merges to exit in one canal; (4) type IV: two separate and distinct canals are present from the pulp chamber to the apex; (5) type V: single canal leaving the pulp chamber but dividing into two separate canals with two separate apical foramina; (6) type VI: two separate canals leave the pulp chamber but join at the midpoint and divides again into two separate canals with two separate apical foramina; (7) Type VII: One canal leave the pulp chamber which divide and again unite into in its course and finally divide into two before exiting from apex; and (8) type VIII: Three canals leave the pulp chamber and run independently towards the apex (Figure 4) [1] [5] [6].

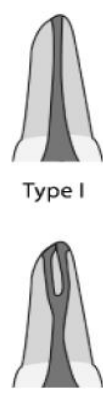

Type V

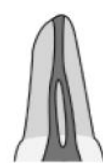

Type ॥

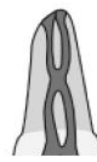

Type VI

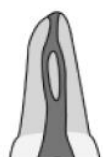

Type III

Type VII

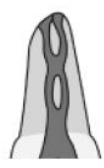



Type IV

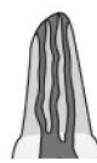

Type VIII
Figure 4. Diagrammatic representation of Vertucci's root canalconfigurations. [4].

Poor cleaning and shaping, and not fully obturated root canal will cause the exixtence of necrotic tissues which will be a failure factor of root canal treatment. Therefore, proper preparation, obturation techniques and adequate medicaments are needed. In this case a crown down preparation technique was carried out using a rotary instrument. Crown down preparation technique starts from coronal to apical with the largest diameter instrument to the smallest diameter [7]. The advantages of rotary instruments using NiTi files are more concentrated in root canals, lower apical transportation, safer and more efficient than manual methods [8].

The obturation technique carried out in this case is warm vertical condensation. This obturation technique was chosen because better root canal closure in the apical, lateral and root canal accessories, produce consistent obturation, stable dimension, fills the irregularity of root canal in three dimension better compared to cold gutta percha technique [2] [9]. The things should be considered of warm vertical condensation technique are the root canal must be a funnel shape, should has apical constriction and constant pressure while condensing through the root canal [9].

Intracanal dressing between visits was needed. In this case calcium hydroxide as root canal medicament was chosen. Calcium hydroxide was chosen because the high $\mathrm{pH}$ value of calcium hydroxide has an effective antibacterial effect. The antibacterial activity of calcium hydroxide occurs when hydroxyl ions contact with the root canal fluid. Hydroxyl ions are oxidants that stabilize the free radicals that react to biomolecules. The mechanism of actions of calcium hydroxide are damaging the bacterial cytoplasmic membrane, denaturing proteins, and damaging bacterial DNA [10].

Proper restoration is necessary to support the success of root canal treatment. In this case, after root canal treatment was performed, the tooth was restored with Class II composite resin restoration with short fiber reinforced composite reinforcement. This restoration was chosen because the post treated tooth was lack of hard tissue, so that a strong restoration was needed. Composite resins have been proven to be aesthetically satisfying. Short fiber reinforced composites contain unique fiber compositions and polymers, thus improving the mechanical and physical properties of the restoration. Composite resin restoration with short fiber reinforced composite reinforcement is an alternative of direct restoration recommended for coronal restorations of large cavities in high pressure areas, especially post treated tooth [11].

\section{CONCLUSION}

The complex anatomy of the root canal should be thoroughly understood for proper management, better diagnosis that will lead to the successful root canal treatment. Root canal treatment with warm vertical condensation obturation technique is the appropriate 
treatment of choice for Vertucci type $\mathrm{V}$ root canal configuration.

\section{REFERENCES}

[1] Awawdeh, L., Abdullah, H. and Al-Qudah, A., 2008, Root Form and Canal Morphology of Jordanian Maxillary First Premolars, JOE, 34 (8)

[2] Hemanth, S., Sujatha, I., Vamshi Krishna, V. and Jayalakshmi, K., 2017, Endodontic Management of Mandibular Second Premolar Having Type-V Vertucci Canal Configuration - Case Series, Saudi J. Oral. Dent. Res., 2 (3) : $76-80$

[3] Vertucci, F. J., 2005, Root Canal Morphology and Its Relationship to Endodontic Procedures, Endodontic Topics, $10: 3-29$

[4] Kulkarni, G., 2017, New Root Canal Obturation Techniques: A Review, EC Dental Science, 11 (2) : 68-76.

[5] Bansal, R., Hegde, S. and Astekar, M. S., 2018 Classification of Root Canal Configurations: A Review and a New Proposal of Nomenclature System for Root Canal Configuration Review Article, Journal of Clinical and Diagnostic Research, 12(5): 1-5

[6] Hepsenoglu, Y. E. and Ersahan, S., 2018, Endodontic Treatment of A Mandibular
Premolar with Vertucci Type V Root Canal Morphology: A Rare Case Report, Turk Endod J., 3(1) : 19-22.

[7] Gallottini, L., 2017, Shaping of the Root Canal System: A Multistep Technique, The Journal of Contemporary Dental Practice, 18(9):851-855

[8] Bahrololoomi, Z., Tabrizizadeh, M. and Salmani, L., 2007, In vitro Comparison of Instrumentation Time and Cleaning Capacity between Rotary and Manual Preparation Techniques in Primary Anterior Teeth, Journal of Dentistry, Tehran University of Medical Sciences; 4.

[9] Garg, N. and Garg. A., 2014, Textbook of Endodontics, ed. 3, Unipress Publishing, Selangor Darul Ehsan

[10] Kim, D. and Kim, E., 2014, Antimicrobial Effect of Calcium Hydroxide as An Intracanal Medicament in Root Canal Treatment: A Literature Review - Part I. In vitro Studies, Restor Dent Endod., 39(4): 241-252.

[11] Garoushi, S., Gargoum, A., Vallittu, P. K. and Lassila, L., 2018, Short Fiber-reinforced Composite Restorations: A review of The Current Literature, J Invest Clin Dent., https://doi.org/10.1111/jicd.12330. 